PROCEEDINGS OF THE

AMERICAN MATHEMATICAL SOCIETY

Volume 128, Number 12, Pages 3483-3492

S 0002-9939(00)05912-8

Article electronically published on August 17, 2000

\title{
EXISTENCE-UNIQUENESS AND LONG TIME BEHAVIOR FOR A CLASS OF NONLOCAL NONLINEAR PARABOLIC EVOLUTION EQUATIONS
}

\author{
AZMY S. ACKLEH AND LAN KE \\ (Communicated by David S. Tartakoff)
}

\begin{abstract}
We establish existence and uniqueness of solutions for a general class of nonlocal nonlinear evolution equations. An application of this theory to a class of nonlinear reaction-diffusion problems that arise in population dynamics is presented. Furthermore, conditions on the initial population density for this class of problems that result in finite time extinction or persistence of the population is discussed. Numerical evidence corroborating our theoretical results is given.
\end{abstract}

\section{INTRODUCTION}

In this paper we consider the following class of nonlinear nonlocal evolution equations on the Banach space $B$ :

$$
\left\{\begin{array}{l}
\dot{u}(t)=\frac{1}{a(u(t))} A u(t)+F(u(t)), \quad t>0, \\
u(0)=u_{0} .
\end{array}\right.
$$

Here, the linear operator $A$ is assumed to be an infinitesimal generator of a $\mathrm{C}_{0}$ semigroup $T(t)$ on $B$, with domain $D(A) \subset B$. The reaction function $F: B \rightarrow$ $B$ is locally Lipschitz continuous in $u$ satisfying $F(0)=0$. The functional $a$ : $B \rightarrow[0,+\infty)$ is assumed to be locally Lipschitz continuous in $u$. Furthermore, we assume $a(\xi)>0$ for all $\xi \neq 0$, and $a(0) \geq 0$. Our first goal is to establish existence-uniqueness of solutions to (1.1). By a solution we mean a $B$-valued function $u(t)$ that satisfies the following:

i) $u(t)$ is continuous for $t \geq 0$ and continuously differentiable for $t>0$.

ii) $u(t) \in D(A)$ for $t>0$.

iii) $u(t)$ satisfies (1.1).

Our motivation for investigating (1.1) arises from reaction-diffusion equations that model population dynamics. For example, the following equation that describes the density of a population subject to spreading falls under the class of

Received by the editors July 20, 1998.

2000 Mathematics Subject Classification. Primary 35K50, 35K55, 35K99, 35B40, 92D25.

Key words and phrases. Nonlocal parabolic evolution equations, unbounded diffusion, population dynamics, asymptotic behavior, extinction, persistence. 
evolution equations presented in (1.1):

$$
\begin{cases}u_{t}=\frac{1}{a(u(t, \cdot))} \Delta u+F(u) & \text { in } \Omega \times(0, T), \\ u(t, x)=0 & \text { on } \partial \Omega \times(0, T), \\ u(x, 0)=u_{0}(x) & \text { on } \bar{\Omega} .\end{cases}
$$

Here $\Omega$ is a bounded open subset in $\mathbb{R}^{n}, n \geq 1$, with smooth boundary $\partial \Omega$. The diffusion coefficient $1 / a$ then depends on a nonlocal quantity related to the total population in the domain, i.e., the diffusion of individuals is guided by the global state of the population in the medium. If the coefficient $1 / a$ is an unbounded function around the origin (e.g., $\left.a(u(t, \cdot))=\int_{\Omega} u(t, x) d x\right)$, then a diffusion of this type could model a population that is anxious to move quickly out of zones experiencing a sharp decrease in population densities (see [7]). For example, consider a population attempting to leave a spatial region due to a sudden dangerous situation. The individuals in the population move randomly (due to lack of information) in an attempt to leave the area. In this case, diffusion out of the region will increase as population decreases due to a decrease in the interaction between individuals that hinders their movement out. One can imagine such an occurrence related to an epidemic (see [7]). The function $F$ describes the reaction or growth of the population. Two commonly used forms for $F$ in the literature are the logistic equation $F(u)=r u(k-u)$ and the Monod kinetics $F(u)=r u /(k+u)$ (see, e.g., 6, 9, 10, 12]).

Local evolution equations of type (1.1) (i.e., $a(u(t)) \equiv 1$ ) have been extensively studied in the literature (see, e.g., [1 2, 3, 4, 5, 8, 9, 11, 13, and the many references therein). Existence-uniqueness of solutions for the local linear and semilinear problems of type (1.1) has been discussed in 1, 9, 11, 13, while in [2, 3, 4, 15, 8 , these results were extended to the nonlinear local case (where $A$ is assumed to be a nonlinear operator). In [7, a homogeneous nonlocal case of type (1.1) (i.e., $F(u(t))=0$ ) was discussed. Therein existence and uniqueness of solutions were established. Our first goal in this paper is to extend these results to the setting presented in (1.1).

This paper is organized as follows. In Section 2, a local existence-uniqueness result for (1.1) is established. Section 3 is devoted to the study of a class of reaction-diffusion problems which falls under the class of equations presented in (1.1). Conditions on the initial population density which result in the population finite time extinction or persistence for all times is established. Finally, in Section 4 we develop a finite difference scheme to approximate the solution of a nonlocal quasilinear reaction-diffusion equation. This scheme is then used to numerically study the long time behavior of the quasilinear model.

\section{ExistenCe-Uniqueness of SOLUTIONS}

We start this section by introducing the following auxiliary nonlocal semilinear evolution equation:

$$
\left\{\begin{array}{l}
\dot{v}(t)=A v(t)+a(v(t)) F(v(t)), \quad t>0, \\
v(0)=u_{0} .
\end{array}\right.
$$

Using (2.1), we can show the following:

Theorem 2.1. For $u_{0} \in D(A)$ with $u_{0} \neq 0$, assume that there exists a positive constant $T_{\max }$ such that (2.1) has a unique solution $v$ on $\left[0, T_{\max }\right)$. Then there 
exists a unique solution $u$ to (1.1) on the interval $\left[0, \bar{T}_{\max }\right)$, where $\bar{T}_{\max }$ is given by

$$
\bar{T}_{\max }=\int_{0}^{T_{\max }} a(v(s)) d s .
$$

Proof. Let $\tau=\tau(t)$ be the solution to the ordinary differential equation

$$
\left\{\begin{array}{l}
\dot{\tau}(t)=\frac{1}{a(v(\tau(t)))}, \quad t>0, \\
\tau(0)=0 .
\end{array}\right.
$$

Separating variables and integrating in $t$ we get the following equation:

$$
\int_{0}^{\tau(t)} a(v(s)) d s=t, \quad t \in\left[0, T_{\max }\right) .
$$

Setting

$$
G(\xi)=\int_{0}^{\xi} a(v(s)) d s,
$$

it can be easily shown that $G$ is a $C^{1}$ diffeomorphism from $\left[0, T_{\max }\right)$ onto $\left[0, \bar{T}_{\max }\right)$. Hence, (2.2) has a unique solution given by $\tau(t)=G^{-1}(t)$ on $\left[0, \bar{T}_{\max }\right)$.

Now let

$$
u(t)=v(\tau(t)) .
$$

Then clearly the $B$-valued function $u$ satisfies the following: $u(0)=v(\tau(0))=u_{0}$ and $u(t)$ is continuous for $t \geq 0$, continuously differentiable and $u(t) \in D(A)$ for $t>0$. Furthermore, we have that

$$
\begin{aligned}
\dot{u}(t) & =\dot{v}(\tau(t)) \dot{\tau}(t)=(A v(\tau(t))+a(v(\tau(t))) F(v(\tau(t)))) \frac{1}{a(v(\tau(t)))} \\
& =\frac{1}{a(u(t))} A u(t)+F(u(t)), \quad t>0 .
\end{aligned}
$$

Hence, $u$ is a local solution of equation (1.1). Conversely let $u$ be a local solution to (1.1) and let $G$ be the solution to the differential equation

$$
\left\{\begin{array}{l}
\dot{G}(s)=a(u(G(s))), \quad t>0, \\
G(0)=0 .
\end{array}\right.
$$

Set

$$
v(t)=u(G(t))
$$

We have that $v(0)=u_{0}, v(t)$ is continuous for $t \geq 0$, continuously differentiable and $v(t) \in D(A)$ for $t>0$. Moreover,

$$
\dot{v}(t)=\dot{u}(G(t)) \dot{G}(t)=A v(t)+a(v(t)) F(v(t)), \quad t>0 .
$$

From (2.4) we can show that $G$ is given by

$$
G(t)=\int_{0}^{t} a(v(s)) d s
$$

and thus $G(\tau(t))=t$. This shows that $u$ is necessarily given by (2.3) and the proof is complete. 
Remark 2.2. Under the Lipschitz continuity requirement on the functions $F$ and $a$ discussed in the previous section, (2.1) has a unique mild solution, i.e., a solution that satisfies the integral equation

$$
v(t)=T(t) u_{0}+\int_{0}^{t} T(t-s) \mathcal{F}(v(s)) d s
$$

for $t \in\left[0, T_{\max }\right)$, where the mapping $\mathcal{F}: B \rightarrow B$ is defined by $\mathcal{F}(v)=a(v) F(v)$. In general, a sufficient condition for this mild solution to be a solution of (2.1) (i.e., to satisfy i)-iii) given in Section 1 ) is for the mapping $\mathcal{F}$ to be continuously differentiable from $B$ into $B$ (see Chapter 6 in [11]). However, in the case $T(t)$ is an analytic semigroup, then (2.1) has a unique solution for $t \in\left[0, T_{\max }\right)$, provided that $\mathcal{F}$ is locally Lipschitz continuous only ([13], page 39 ).

\section{Finite time extinction and persistence FOR A CLASS OF PARABOLIC EQUATIONS}

Choose the Banach space $B=L^{2}(\Omega)$, where $\Omega$ is a bounded region in $\mathbb{R}^{n}$ with smooth boundary $\partial \Omega$. Let $L$ be a uniform linear elliptic operator given by

$$
L u=\sum_{i=1}^{n} \frac{\partial}{\partial x_{i}}\left(\sum_{j=1}^{n} a_{i j}(x) \frac{\partial u}{\partial x_{j}}\right)
$$

with sufficiently smooth and symmetric coefficients $a_{i j}(x)$, and consider the following class of reaction-diffusion equations:

$$
\begin{cases}u_{t}=\frac{1}{\int_{\Omega} u(t, x) d x} L u+f(u) & \text { in } \Omega \times\left(0, \bar{T}_{\max }\right), \\ u=0 & \text { on } \partial \Omega \times\left(0, \bar{T}_{\max }\right), \\ u(0, x)=u_{0}(x) & \text { on } \bar{\Omega},\end{cases}
$$

with

$$
f(u)=\frac{r u}{k+u} \text { or } f(u)=r u(k-u),
$$

where $r$ and $k$ are constants. For the rest of this section, we assume that the initial population density $u_{0} \in C_{0}^{2}(\Omega)$ satisfying $u_{0} \geq 0$ and $u_{0} \neq 0$. Our goal is to find a function $\underline{u}_{0}(x)$ such that if $u_{0}(x)<\underline{u}_{0}(x)$, then the population goes to extinction in finite time (i.e., the solution to equation (3.1) satisfies $\lim _{t \rightarrow \bar{T}_{\max }} \int_{\Omega} u(t, x)=0$ ) and a function $\bar{u}_{0}(x)$ such that if $u_{0}(x) \geq \bar{u}_{0}(x)$, then the population persists (i.e., $\left.\liminf _{t \rightarrow \infty} \int_{\Omega} u(t, x)>0\right)$.

With the second order differential operator $L$ we associate the operator $A$ on $L^{2}(\Omega)$ as follows:

a) The domain of definition $D(A)=H^{2}(\Omega) \cap H_{0}^{1}(\Omega)$.

b) $A u=L u$ for $u \in D(A)$.

It is well known that the linear operator $A$ is an infinitesimal generator of an analytic semigroup of contractions $T(t)$ on $L^{2}(\Omega)$ (e.g., [11, page 215).

3.1. Finite time extinction. Let $\phi(x)$ be the first eigenfunction of

$$
\begin{cases}-L \phi=\lambda \phi & \text { in } \Omega \\ \phi=0 & \text { on } \partial \Omega\end{cases}
$$


with $\|\phi\|_{\infty}=1$, and let $\lambda_{0}$ be the first eigenvalue. Recall that $\phi$ is a positive function in $\Omega$. For the case $f(u)=r u /(k+u)$, defining $\underline{u}_{0}(x)=\frac{\lambda_{0}}{c} \phi$ where $c=\frac{r}{k} \int_{\Omega} \phi d x$ we have the following theorem:

Theorem 3.1. If $u_{0}(x)<\underline{u}_{0}(x)$, then the solution $u$ to equation (3.1) goes to extinction in finite time.

Proof. Define $F(v)=\max \{r v /(k+v), 0\}$ and $a(v(t))=\int_{\Omega}|v(t, x)| d x$, and consider the following semilinear problem:

$$
\begin{cases}v_{t}=L v+a(v(t)) F(v(t)) & \text { in } \Omega \times\left(0, T_{\max }\right), \\ v=0 & \text { on } \partial \Omega \times\left(0, T_{\max }\right), \\ v(0, x)=u_{0}(x) & \text { on } \bar{\Omega} .\end{cases}
$$

Clearly, $F$ and $a$ defined above are locally Lipschitz continuous. Hence, rewriting (3.2) as an evolution equation of the form (2.1) and using Remark 2.2 we get that there exists a unique solution to equation (3.2) on the interval $\left[0, T_{\max }\right)$, for some $T_{\max }>0$. Since the initial density $u_{0}$ is twice continuously differentiable and satisfies the boundary condition, the comparison principle discussed in [12], Section 2.7, applies to (3.2). Hence, we start by constructing upper and lower solutions to (3.2).

Clearly the zero function is a lower solution to equation (3.2). Next, we construct an upper solution, $\bar{v}$, to equation (3.2) of the form

$$
\bar{v}=p(t) \phi(x) \text {. }
$$

Then

$$
\bar{v}_{t}-L \bar{v}=\left(\dot{p}+\lambda_{0} p\right) \phi .
$$

Hence, $\bar{v}$ is an upper solution provided that

$$
p(0) \phi(x) \geq u_{0}(x)
$$

and

$$
\left(\dot{p}+\lambda_{0} p\right) \phi \geq \int_{\Omega} \bar{v}(t, x) d x \frac{r p \phi}{k+p \phi} .
$$

Since

$$
\int_{\Omega} \bar{v}(t, x) d x \frac{r p \phi}{k+p \phi} \leq p \int_{\Omega} \phi d x \frac{r p \phi}{k+p \phi} \leq p^{2} \int_{\Omega} \phi d x \frac{r \phi}{k},
$$

then (3.3) is true if the following equality holds:

$$
\dot{p}+\lambda_{0} p=p^{2} \int_{\Omega} \phi d x \frac{r}{k} .
$$

Solving for $p$ we get

$$
p(t)=\frac{\lambda_{0} p(0) e^{-\lambda_{0} t}}{\lambda_{0}-c p(0)+c p(0) e^{-\lambda_{0} t}}
$$

where $c$ is defined above.

Hence, if $p(0)=\alpha<\lambda_{0} / c$, then $p(t)$ exists globally and approaches zero as $t \rightarrow \infty$. Therefore, if the initial condition of the population is small enough such that

$$
u_{0}(x)<\frac{\lambda_{0}}{c} \phi=\underline{u}_{0}(x)
$$


then (3.2) has a global classical solution $v$ (see Theorem 7.1, page 83 in [12]). It is not too difficult to argue that this solution coincides with the semigroup solution of Remark 2.2. Furthermore, $v$ satisfies the following:

$$
0<v(t) \leq \frac{\lambda_{0} \alpha e^{-\lambda_{0} t} \phi}{\lambda_{0}-c \alpha+c \alpha e^{-\lambda_{0} t}}, \quad t>0
$$

Hence, $v(t) \rightarrow 0$ as $t \rightarrow \infty$. Applying Theorem 2.1, we get that

$$
\bar{T}_{\max }=\int_{0}^{\infty} a(v(s)) d s=\int_{0}^{\infty} \int_{\Omega} v(s, x) d x d s \leq \int_{0}^{\infty} \frac{\lambda_{0} \alpha e^{-\lambda_{0} t} \int_{\Omega} \phi d x}{\lambda_{0}-c \alpha+c \alpha e^{-\lambda_{0} t}} d t<\infty .
$$

From the nonnegativity of $v$, the solution to (3.1) satisfies

$$
u(t)=v(\tau(t)) \rightarrow 0, \text { as } t \rightarrow \bar{T}_{\max }
$$

This establishes the desired result.

For the case $f(u)=r u(k-u)$, we set $\underline{u}_{0}(x)=\frac{\lambda_{0}}{c} \phi$ where $c=r k \int_{\Omega} \phi d x$. Then we have the following theorem:

Theorem 3.2. If $u_{0}(x)<\underline{u}_{0}(x)$, then the solution $u$ to equation (3.1) goes to extinction in finite time.

Proof. Similar arguments as those in the proof of Theorem 3.1 establish the result.

3.2. Persistence. Let us consider first the case $f(u)=r u /(k+u)$. Assume that $r \int_{\Omega} \phi d x>\lambda_{0}$, set

$$
\delta=\frac{k \lambda_{0}}{r \int_{\Omega} \phi d x-\lambda_{0}}
$$

and define $\bar{u}_{0}(x)=\delta \phi$ where $\phi$ and $\lambda_{0}$ are the same as before. Then we have the following:

Theorem 3.3. If $u_{0}(x) \geq \bar{u}_{0}(x)$, then the solution $u$ to equation (3.1) is persistent.

Proof. Clearly $p(t)=p(0) e^{|\Omega| t}$ with $p(0) \geq\left\|u_{0}\right\|_{\infty}$ is an upper solution to (3.2). Furthermore, letting $\hat{v}=\delta \phi$, then using similar arguments as above it can be easily verified that $\hat{v}$ is a lower solution to (3.2), provided that $u_{0} \geq \delta \phi$. Hence, we see that a classical solution $v(t)$ to equation (3.2) exists globally and for $u_{0} \geq \delta \phi$ we have

$$
v(t) \geq \hat{v}=\delta \phi
$$

By a similar argument as in the proof of Theorem 3.1 we can apply Theorem 2.1 to obtain

$$
\bar{T}_{\max }=\int_{0}^{\infty} a(v(s)) d s=\int_{0}^{\infty} \int_{\Omega} v(s, x) d x d s=\infty .
$$

Hence the solution to (3.1) satisfies

$$
u(t)=v(\tau(t)) \geq \delta \phi .
$$

This establishes the desired result. 
For the case $f(u)=r u(k-u)$, assume that $4 \lambda_{0}<r k^{2} \int_{\Omega} \phi d x$, let $\delta$ be a positive solution of

$$
\lambda_{0}=r(k-\delta) \delta \int_{\Omega} \phi d x
$$

and define $\bar{u}_{0}(x)=\delta \phi$. Then we have the following:

Theorem 3.4. If $u_{0}(x) \geq \bar{u}_{0}(x)$, then the solution $u$ to equation (3.1) is persistent.

Proof. For this case, an upper solution to (3.2) can be easily constructed as $\bar{v}=M$ where $M=\max \left\{k,\left\|u_{0}\right\|_{\infty}\right\}$. Using similar arguments as in the proof of Theorem 3.3 we can show that $\hat{v}=\delta \phi$ is a lower solution to (3.2). Therefore, we obtain that (3.2) has a classical global solution $v(t)$, and the result can then be established by repeating the arguments above.

Remark 3.5. In the proofs of Theorems 3.1-3.4 we establish more than finite time extinction and persistence of the total population. In particular, from the proofs of Theorems 3.1-3.2 we conclude that

$$
\lim _{t \rightarrow \bar{T}_{\max }} \sup _{x \in \Omega} u(t, x)=0 .
$$

Furthermore, from the proofs of Theorems 3.3-3.4 it follows that for any compact set $Q \subset \Omega$ the solution to (3.1) satisfies liminf $t \rightarrow \infty u(t, x)>0$, for all $x \in Q$.

Remark 3.6. The results of this section can be extended to the following setting:

$$
\begin{cases}u_{t}=\frac{1}{b\left(\int_{\Omega} u(t, x) d x\right)} L u+f(u) & \text { in } \Omega \times\left(0, \bar{T}_{\max }\right), \\ u=0 & \text { on } \partial \Omega \times\left(0, \bar{T}_{\max }\right), \\ u(0, x)=u_{0}(x) & \text { on } \bar{\Omega}\end{cases}
$$

with $b$ being an increasing continuously differentiable function with $\left|\frac{d b}{d \xi}(\xi)\right| \leq \beta$, for all $\xi \geq 0$, and $b(0)=0$.

\section{Numerical RESUlts}

In this section we consider the following special case of equation (3.1):

$$
\begin{cases}u_{t}=\frac{1}{\int_{0}^{1} u(t, x) d x} u_{x x}+r u(k-u), & (x, t) \in(0,1) \times\left(0, \bar{T}_{\max }\right), \\ u(t, 0)=0=u(t, 1), & t \in\left(0, \bar{T}_{\max }\right), \\ u(0, x)=u_{0}(x), & x \in[0,1] .\end{cases}
$$

To numerically solve this equation the following implicit backward finite difference approximation was employed:

$$
\begin{cases}\frac{u_{j}^{i+1}-u_{j}^{i}}{\Delta t}=\frac{1}{\sum_{j=1}^{N} \Delta x u_{j}^{i}} \frac{u_{j+1}^{i+1}-2 u_{j}^{i+1}+u_{j-1}^{i+1}}{\Delta x^{2}}+r u_{j}^{i}\left(k-u_{j}^{i}\right), & j=1, \ldots, N-1, \\ & i=1, \ldots, M \\ u_{0}^{i+1}=0=u_{N}^{i+1}, & i=1, \ldots, M \\ u_{j}^{0}=u_{0}\left(x_{j}\right), & j=0, \ldots, N\end{cases}
$$

where $\Delta t=\bar{T}_{\max } / M, \Delta x=1 / N, x_{j}=j \Delta x, j=0, \ldots, N$, and $t_{i}=i \Delta t$, $i=0, \ldots, M$. In (4.2) $u_{j}^{i}$ denotes the difference approximations of $u\left(t_{i}, x_{j}\right)$. The time $\bar{T}_{\max }$ is determined in our simulation as the time that satisfies the stopping 
criteria $u_{j}^{M}<\epsilon$, where $\epsilon$ is a given tolerance. Hence, solving the discrete system (4.2) is equivalent to solving the following tridiagonal system of linear equations:

$$
A^{i} \vec{u}^{i+1}=\vec{f}^{i} \text { for } i=1, \ldots, M,
$$

where

$$
\begin{aligned}
& A^{i}=\left(\begin{array}{llllll}
\left(1+\frac{2 \mu}{P^{i}}\right) & \left(-\frac{\mu}{P^{i}}\right) & 0 & 0 & \ldots & 0 \\
\left(-\frac{\mu}{P^{i}}\right) & \left(1+\frac{2 \mu}{P^{i}}\right) & \left(-\frac{\mu}{P^{i}}\right) & 0 & \ldots & 0 \\
0 & \left(-\frac{\mu}{P^{i}}\right) & \left(1+\frac{2 \mu}{P^{i}}\right) & \left(-\frac{\mu}{P^{i}}\right) & \ldots & 0 \\
\ldots & \ldots & \ldots & \ldots & \ldots & \ldots \\
0 & \ldots & 0 & \left(-\frac{\mu}{P^{i}}\right) & \left(1+\frac{2 \mu}{P^{i}}\right) & \left(-\frac{\mu}{P^{i}}\right) \\
0 & \ldots & 0 & 0 & \left(-\frac{\mu}{P^{i}}\right) & \left(1+\frac{2 \mu}{P^{i}}\right)
\end{array}\right), \\
& \mu=\frac{\Delta t}{(\Delta x)^{2}}, \vec{u}^{i+1}=\left[u_{1}^{i+1}, u_{2}^{i+1}, \ldots, u_{N-1}^{i+1}\right], P^{i}=\sum_{j=1}^{N-1} u_{j}^{i} \Delta x, \text { and } \\
& \vec{f}^{i}=\left[u_{1}^{i}+\Delta \operatorname{tr} u_{1}^{i}\left(k-u_{1}^{i}\right), u_{2}^{i}+\Delta \operatorname{tr} u_{2}^{i}\left(k-u_{2}^{i}\right)\right. \\
& \left., \ldots, u_{N-1}^{i}+\Delta t r u_{N-1}^{i}\left(k-u_{N-1}^{i}\right)\right] .
\end{aligned}
$$

In all of our simulations presented in this section we set $r=1, k=10$ and $\epsilon=10^{-5}$.

Recall that the first eigenvalue and corresponding eigenfunction of

$$
\left\{\begin{aligned}
-\phi^{\prime \prime} & =\lambda \phi \\
\phi(0) & =0=\phi(1)
\end{aligned} \quad \text { in }(0,1),\right.
$$

is given by $\lambda_{0}=\pi^{2}$ and $\phi=\sin \pi x$. Hence, for equation (4.1) $c=r k \int_{0}^{1} \sin \pi x d x \approx$ 6.4 and $\underline{u}_{0}(x)=\frac{\pi^{2}}{c} \sin \pi x \approx 1.55 \sin \pi x$. In our numerical simulations, we set $u_{0}(x)=1.5 \sin \pi x$ and present the results in Figure 1. The simulation indicates that extinction occurs at $\bar{T}_{\max }=0.32$ for this case. From (3.5) simple calculations reveal that an upper bound on $\bar{T}_{\max }$ is 0.34 which is in agreement with the numerical result presented in Figure 1.

As for persistence, we choose $\bar{u}_{0}(x)=\delta \sin \pi x$, where $\delta=1.92$ is the first positive solution of equation (3.6). Hence, for $u_{0}(x) \geq \bar{u}_{0}(x)$ the solution should persist for all times. Figure 2 presents the result of our simulation with $u_{0}(x)=$ $1.95 \sin \pi x$. This simulation indicates that the population converges to a positive steady state.

Finally, we mention that we have performed numerical simulations for initial conditions $1.55 \sin \pi x \leq u_{0}(x)<1.92 \sin \pi x$. While our theoretical results do not conclude what the long time behavior is for such initial conditions, simulation results indicate that extinction occurs for all $u_{0}(x)<1.84 \sin \pi x$. However, if $u_{0}(x) \geq 1.84 \sin \pi x$, then the population persists. In fact, it tends to a positive steady state. 


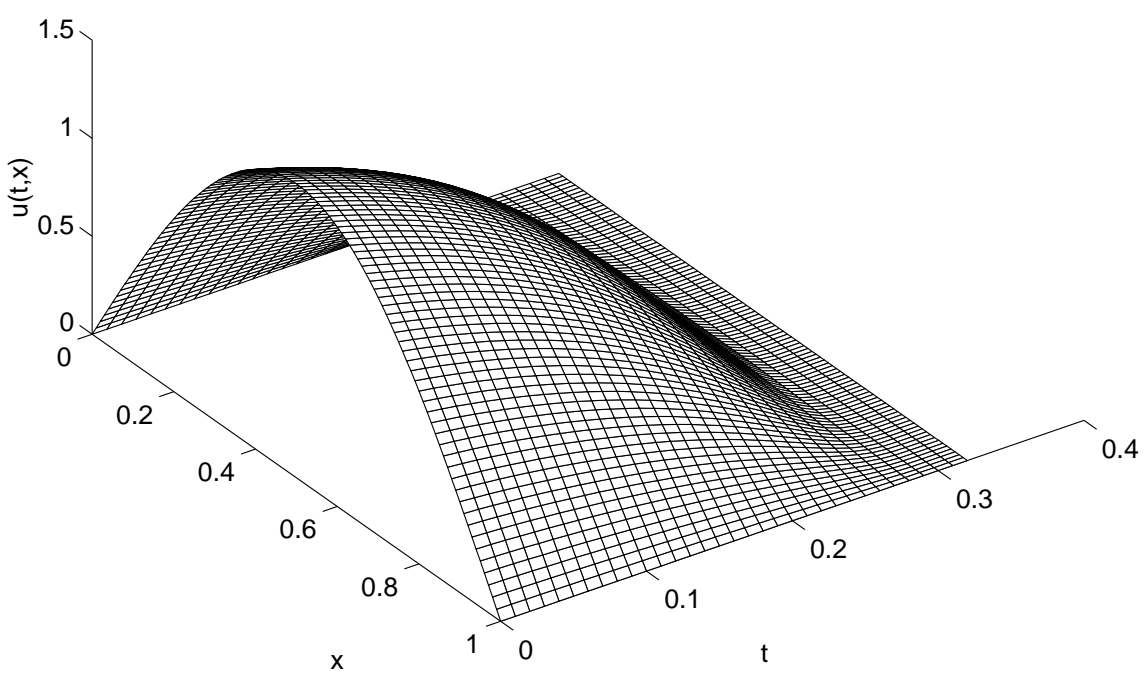

Figure 1. Extinction of the population density $u(t, x)$ at time $\bar{T}_{\max }=0.32$.

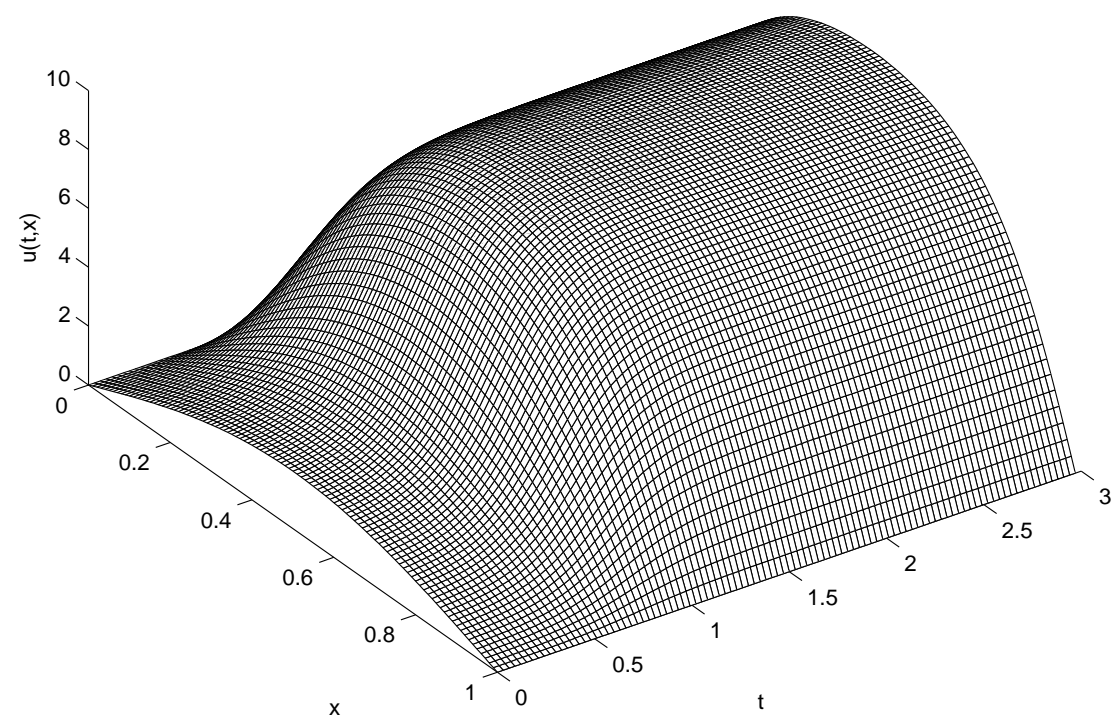

Figure 2. Persistence of the population density $u(t, x)$. 


\section{ACKNOWLEDGMENTS}

The authors would like to thank an anonymous referee for useful comments and suggestions. They would also like to thank Robert Ferdinand for programming support. The research of the first author was supported by the Louisiana Education Quality Support Fund under grant \# LEQSF(1996-99)-RD-A-36.

\section{REFERENCES}

[1] A. S. Ackleh and B. G. Fitzpatrick, Estimation of Time Dependent Parameters in general Parabolic Evolution Systems, Journal of Mathematical Analysis and Applications, 203 (1996), 464-480. MR 97f:34040

[2] A. S. Ackleh and S. Reich, Parameter Estimation in Nonlinear Evolution Equations, Numerical Functional Analysis and Optimization, 19 (1998), 933-947. MR 99k:93038

[3] H. T. Banks, S. Reich and I. G. Rosen, An Approximation Theory for the Identification of Nonlinear Distributed Parameter Systems, SIAM Journal on Control and Optimization, 28 (1990), 552-569. MR 91g:47051

[4] H. T. Banks, S. Reich and I. G. Rosen, Galerkin Approximation for Inverse Problems for Nonautonomous Nonlinear Distributed Systems, Applied Mathematics and Optimization, 24 (1991), 233-256. MR 92i:65102

[5] V. Barbu, Nonlinear Semigroups and Differential Equations in Banach Spaces, Nordhoff, Leyden, 1976. MR 52:11666

[6] J. Bear, Dynamics of Fluids in Porous Media, Elsevier, New York, 1972.

[7] M. Chipot and B. Lovat, Existence and Uniqueness Results for a Class of Nonlocal Elliptic and Parabolic Problems, Dynamics of Discrete Continuous and Impulsive Systems, to appear. Manuscript can be obtained from internet: http://www.math.unizh.ch/ ${ }^{\sim}$ chipot/Quenching.ps.

[8] M. G. Crandall and A. Pazy, Nonlinear Evolution Equations in Banach Space, Israel J. Math. 11 (1972), 57-94. MR 45:9214

[9] B. G. Fitzpatrick, Analysis and Approximation for Inverse Problems in Contaminant Transport and Biodegradation Models, Numerical Functional Analysis and Optimization, 16 (1995), 847-866. MR 97g:76088

[10] R. A. Freeze and J. Cherry, Groundwater, Prentice-Hall, Englewodd Cliffs, NJ, 1979.

[11] A. Pazy, Semigroups of Linear Operators and Applications to Partial Differential Equations, Springer-Verlag, New York, 1983. MR 85g:47061

[12] C. V. Pao, Nonlinear Parabolic and Elliptic Equations, Plenum Press, New York, 1992. MR 94c:35002

[13] K. Taira, Analytic Semigroups and Semilinear Initial Boundary Value Problems, Cambridge University Press, New York, 1995. MR 97g:47035

Department of Mathematics, University of Louisiana at Lafayette, Lafayette, LouISIANA 70504-1010

E-mail address: ackleh@louisiana.edu

Department of Mathematics, University of Louisiana at Lafayette, Lafayette, LouISIANA 70504-1010

E-mail address: ke@louisiana.edu 